\title{
Alternatibong Pagkalalaki, Alternatibong Musika: Ang Eraserheads at Kulturang Popular ng Dekada ' 90
}

\author{
Michael D. Pante
}

\begin{abstract}
Abstrakt
Lubhang maimpluwensiya ang bandang Eraserheads sa musikang Filipino, subalit hindi kinikilala ang banda bilang tagapagtaguyod ng progresibong politika. Gayumpaman, sa peministang pagsusuri ng mga kanta ng banda, lalo na yaong mga awitin nilang hindi sumikat, makikita ang isang mayabong na diskurso ng kritisismo sa dominanteng pagpapakahulugan sa pagkalalaki. Bagama't nakilala ang banda sa mga popular na kanta na may malinaw na pagkiling sa heteronormative na depinisyon ng pag-ibig, may mga awitin din silang humuhulagpos mula sa dominanteng pag-unawa sa kasarian. Habang hindi isinasantabi ang isyu ng komersiyalisadong kulturang popular, layunin ng sanaysay na ito na balikan ang mga himig ng Eraserheads bilang pagpapatampok sa alternatibong musika na naglalaman ng alternatibong pagtingin sa pagiging isang lalaki sa kasalukuyang lipunang nasasakal na sa populistang machismo.
\end{abstract}

Keywords: kasarian, musika, OPM, banda, sensura 


\begin{abstract}
The rock band Eraserheads exerts a huge influence on the Philippine music industry, but does not carry a reputation for advocating progressive politics. Nonetheless, by analyzing their songs, especially those that did not enjoy popularity, one sees a rich discourse of critique targeted at the dominant notion of manliness. Although the band is famous for lyrics that favored the heteronormative definition of romantic love, they also have songs that break free from the predominant understanding of gender. Without dismissing the issue of commercialized popular culture, this essay seeks to revisit the Eraserheads' discography to foreground alternative music that showcases alternative perspectives about manliness against the context of present-day Philippine society constricted by macho populism.
\end{abstract}

Keywords: gender, music, OPM, band, censorship

\title{
Plaridel Open Access Policy Statement
}

As a service to authors, contributors, and the community, Plaridel: A Philippine Journal of Communication, Media, and Society provides open access to all its content. To ensure that all articles are accessible to readers and researchers, these are available for viewing and download (except Early View) from the Plaridel journal website, provided that the journal is properly cited as the original source and that the downloaded content is not modified or used for commercial purposes. Plaridel, published by the University of the Philippines College of Mass Communication is licensed under Creative Commons Attribution-NonCommercial-NoDerivatives 4.0 International License (https://creativecommons.org/ licenses/by-nc-nd/4.0/legalcode).

\section{How to cite this article in APA}

Pante, M. D. (2021). Alternatibong pagkalalaki, alternatibong musika: Ang Eraserheads at kulturang popular ng dekada '90. Plaridel, 18(1), 1-29. https://doi.org/10.52518/2020-05pante 


\section{Pasakalye Papuntang Dekada Nobenta}

Pinag-usapan nang husto ang konsepto ng "toxic masculinity" nitong mga nagdaang taon. Naging prominente ito lalo na sa social media dahil sa mga dumaraming kaso ng pambabastos at iba pang anyo ng paglabag sa karapatan ng kababaihan sa iba't ibang panig ng daigdig. Kaalinsabay nito ang pagiging popular ng mga kampanya gaya ng \#MeToo at \#BabaeAko. Marahil, naging mabigat ang halagang mapag-usapan ang "toxic masculinity" dulot na rin ng pagkakaroon ng mga prominenteng halimbawa nito sa publikong diskusyon, mula sa mga lider-pambansa, gaya nina Donald Trump at Rodrigo Duterte, hanggang sa mga personalidad sa midya, tulad nina Kevin Spacey at ang magkakapatid na Tulfo.

Kasama ang Pilipinas sa malawakang diskursong ito na layong ituon ang atensiyon ng karahasang seksuwal palayo sa kababaihan at tungo sa kalalakihan at ang mga estrukturang panlipunan na pumapabor sa dominanteng kasarian. Gayumpaman, tila naninibago pa rin ang lipunang Filipino sa ganitong direktang atake sa pagkalalaki, lalo pa't nasanay ang karamihan na sisihin ang mga diumanong maling kilos ng kababaihan (hal., pananamit, pananalita, pagpunta sa delikadong lugar, atbp.) o sa diumanong abnormalidad na ikinakabit sa mga bakla at lesbiyana. Isang malaking hamon pa rin ang pagbaligtad sa mga ganitong atrasadong nosyon, at layunin ng papel na ito na magpanukala ng isang alternatibong pananaw: makatutulong sa pagpapayabong sa diskurso tungkol sa toxic masculinity ang pagsusuri sa mga kanta ng isa sa mga pinakamaimpluwensiyang banda sa kasaysayan ng musikang Filipino: ang Eraserheads.

Hindi agad maiuugnay ang Eraserheads sa mga usaping ukol sa kasarian, o anupamang isyung panlipunan. Sumikat ang banda noong dekada '90 dahil sa mga awit na may mga paksang "magaan": romantikong pag-ibig, pagkakaibigan, at mga inosenteng kalokohan ng kabataan. Sa mahabang listahan ng mga popular nilang kanta, mahihirapan ang isang karaniwang tagapakinig na ikabit ang Eraserheads sa progresibong politika gaya ng ilang bandang kasabayan nila tulad ng The Jerks at Yano. Gayumpaman, sa mas masusing paggalugad sa mga di-kilalang kanta ng Eraserheads, malalantad ang malalim na pagpuna ng banda sa dominanteng pagpapakahulugan ng pagkalalaki sa lipunang patriyarkal ng Pilipinas.

Susuriin ng sanaysay na ito ang mga awit ng Eraserheads gamit ang peministang lente. Maraming popular na kanta ang banda, subalit hindi pagtutuunan ng papel ang mga ito. Sa halip, tututok ang pagsusuri sa mga kantang kasama sa mga album nila ngunit hindi pinatugtog sa radyo. Gamit ang metodo ng paghimay sa paglalarawan ng mga persona at karakter na lalaki at babae sa mga liriko, maipapakita ng papel ang malaking pagkakaiba sa diskurso ng kasarian sa mga sikat nilang awitin kompara sa kanilang mga 
di-popular na kanta. Sa pagtatapos, magbibigay ng paunang paliwanag sa likod ng pagkakaibang ito, na nakaangkla sa lohika ng tubo at pagpapasiya ng mga ehekutibong namuhunan para magawang kalakal ang mga kanta ng banda.

Isang dahilan kaya kulang ang pagkilala sa Eraserheads sa mga usaping pangkasarian ay dahil sa kakapusan ng mga akademikong pagaaral tungkol sa banda. Bagama't may mayabong na literatura tungkol sa kontemporanyong musikang popular ng Pilipinas na makikita halimbawa sa mga akda nina Christine Balance (2016), James Gabrillo (2018a; 2018b), at David San Juan (2019), wala sa mga akdang ito ang tuwirang tumitingin sa Eraserheads bilang pangunahing paksa ng pagsusuri. Lubhang kataka-taka ito sa layo na ng naabot ng aralin sa kulturang popular sa Pilipinas, gayong ang banda ang poster boys ng musika ng dekada ' 90 , at napasabak din sa telebisyon, takilya, at pag-eendorso ng mga produkto. Samakatwid, hindi maaaring pag-usapan ang Eraserheads nang labas sa konteksto ng dekadang ito, na madalas na binabansagang post-EDSA o post-authoritarian bilang pagtukoy sa lipunang Filipino matapos ang rehimen ni Ferdinand Marcos (1965-1986) na napatalsik ng EDSA People Power noong 1986.

Maraming balintunang bitbit ang lipunang post-EDSA. Sa isang banda, tila nawasak ang patriyarkal at militarisadong estrukturang tinuntungan ng diktaduryang Marcos. Higit itong napapalitaw kapag ikinompara sa ikonograpiyang pumalit: si Corazon Aquino, ang unang babaeng pangulo ng bansa, at ang Our Lady of EDSA, ang relihiyosong imaheng naging simbolo ng pagbagsak ni Marcos. Sa kabilang banda, lubhang marami din ang mga mapaniil na estrukturang nagpatuloy na puminsala sa lipunang Filipino, mula dekada '70 hanggang '90 kahit nagpalit na ng rehimen (San Juan, 2019). Isa na rito ang pananatili ng konserbatibong pagtingin sa kasarian sa iba't ibang larangan ng midya.

Mapatelebisyon o pelikula, kapos sa espasyo ang kulturang popular ng lipunang post-EDSA para sagkain ang mga pamantayang heteronormative. Sa halip, nagkaroon lang ng mga bagong imahen ng mga tradisyunal na pag-unawa sa kasarian na makikita sa mga popular na palatuntunan. Halimbawa, sa pelikula, bagama't malakas pa rin ang hatak ni Fernando Poe Jr., ang Hari ng Pelikulang Filipino, bilang arketipo ng heteroseksuwal na lalaki, umusbong sa dekada '90 ang mga bagong pangalan, gaya nina Robin Padilla para sa mga pelikulang aksiyon (hal., Anak ni Baby Ama, Bad Boy) at si Richard Gomez (hal., Hihintayin Kita sa Langit, Wating) sa drama (Tolentino, 2000). Ganito rin ang sitwasyon sa telebisyon, kung saan namayagpag ang genre ng sitcom. Halimbawa ng mga ito ang Ober Da Bakod at Palibhasa Lalaki, na pinagbibidahan ni Gomez na gumanap sa bidang karakter na si Ricky. Maihahanay rin dito ang Home Along Da 
Riles, ang sitcom na tumagal nang pinakamahabang panahon sa kasaysayan ng telebisyon sa Pilipinas at pinagbibidahan ng tinaguriang Hari ng Komedya, si Dolphy. Bida sa Home Along Da Riles (Reyes \& Manahan, 1992) ang arketipong uliran-subalit-maralitang ama na si Kevin Cosme bilang katunggali ng isang di-pamilyado, mataray, at matapobreng bading na si Steve (na ginampanan ni Bernardo Bernardo). Dapat ring banggitin na lumaganap ang ganitong malakarikaturang karakterisasyon sa mga bading, mula kay Benny (na ginampanan ni Roderick Paulate) sa programang Abangan ang Susunod na Kabanata (Reyes \& Manahan, 1991) hanggang kay Pao Tang ng seryeng Pugad Baboy, isa sa mga pinakasikat na comic strip ng dekada. Tulad ng arketipo ng lalaking heteroseksuwal, nakaugat sa kulturang popular ng panahong pre-EDSA ang ganitong pagkakahon sa mga bading na kinakatawan ng mga karakter gaya ni Facifica Falayfay. Sa madaling sabi, kulang ang kulturang popular ng dekada '90 sa malalalim na karakterisasyon ng lalaki, heteroseksuwal man o homoseksuwal.

Gayumpaman, ang mga halimbawang nabanggit ay pawang mga representasyong audio-biswal, mga moda ng kulturang popular na nasuri na ng maraming iskolar gamit ang lente ng pagkalalaki (Tolentino, 2000; Cañete, 2014). Panahon na para talakayin naman ito gamit ang anggulo ng pandinig, isang larangang tila hindi pa rin napakikinggan ng marami.

Mayaman ang araling Filipino sa mga akdang sumusuri sa musika at lipunan. Nariyan ang mga akdang pangkasaysayan na madalas nakatutok sa panahong kolonyal, pati na rin ang ethnomusicology, gayundin ang mga sosyologo na layong pulsuhan ang madla batay sa mga pang-arawaraw na himig. Sa usapin ng kontemporanyong musikang popular, hindi maaaring alisin sa diskusyon ang pag-usbong ng Original Pilipino Music (OPM). Nakaugat ang OPM sa Batas Militar (Navarro, 2014), ang pamana ng diktadurya sa lokal na industriya ng musika. Sa orihinal na konseptuwalisasyon nito, may dalawang tila magkasalungat na salik sa paglitaw ng OPM. Sa isang banda, isa itong opisyal na pagpapatampokng mga Filipinong mang-aawit, na lugmok noon sa kompetisyon mula sa dayuhang talento. Sa pamamagitan ng kontrol ng estado sa midya, nagkaroon ng espasyo para sa mga Filipinong mang-aawit at banda, bagama't sa maraming pagkakataon tila nanggagaya pa rin sa Kanluraning estetika (Caruncho, 1996). Pero sa kabilang dako, malinaw din ang ideolohikal na pagkiling ng sistemang ito pabor sa estado. Pawang mga "radio-friendly" na tugtugin ang pinapatampok, na maaaring sabihing lokalisasyon lamang ng pop genre na dominante rin noon. Sa ganitong konteksto sumikat ang tinaguriang Manila Sound, na nakatarget sa kabataang konsumer kung kaya't ang mga liriko (na hindi gumagamit ng purong Ingles o purong Filipino kundi ang "taal" na pananalita sa mga lansangan ng siyudad na paghahalo ng dalawang 
wika, o ang tinaguriang Taglish), maging ang postura ng mga mang-aawit, ay malapit sa karanasan ng tipikal na gitnang-uring tagalungsod sa Pilipinas (Castro, 2011). Higit pa rito, sa mas malalim na pagsusuri, tumutumbas din ang katagang "radio-friendly" sa pagiging "regime-friendly" ng mga awiting hindi tumatahak sa mga larangang lantarang politikal. Ang "pamanang" ito ng rehimeng Marcos ang siyang lunduyan ng kasikatan ng Eraserheads, tagabandera ng OPM sa dekada '90.

Malalim ang naging impluwensiya ng rehimeng Marcos sa kontemporanyong lipunang Filipino, at hindi ito nagtatapos sa usapin ng mga tugtugin sa radyo. Makikita ito maging sa usapin ng kasarian, partikular na ang pagdidiin sa sistemang patriyarkal at pagiging dominante ng ilang partikular na tipo ng pagkalalaki. Anupa't nakasalalay ang rehimen sa lakas militar, bruskong kapatiran, at awtokratikong paternalismo. Dahil rito, susi ang pagpapanatili ng kaayusang heteronormative, sa kabila ng paglala ng krisis ng pagkalalaki na lumitaw sa iba't ibang anyo, gaya na lang ng unang bugso ng sistematikong pagpapadala ng mga migranteng lalaki para maging manggagawa sa Gitnang Silangan (Serquiña, 2019) at ang pagyugyog sa tradisyonal na pag-unawa sa kasarian dulot ng lokal at pandaigdigang aktibismo. Marami-rami na rin ang naisulat tungkol sa relasyon ng batas militar at pagkalalaki, mapaaraling akademiko man (McCoy, 1999; Tolentino, 2012) o mga akdang-panitikan at likhang-sining, gaya ng mga nobelang Dekada '70 at pelikulang Batch '81.

Gayumpaman, muli na namang hindi napapansin ang ugnayan ng pagkalalaki at diktadurya sa larangan ng popular na musika. Sa pamamayagpag ng OPM sa dekada '70 at '80, malinaw rin na walang balak ang industriya na baklasin ang heteronormativity sa pamamagitan ng mga awitin. Hindi hayagang pro-Marcos ang oryentasyong radio-friendly at regime-friendly ng OPM, pero hindi rin nito kukuwestiyunin ang umiiral na kaayusan (Castro, 2011). Iwas-pusoy ang mga temang inilabas sa panahong ito. Isang halimbawa nito ang genre ng ballad na limitado sa mga pampakilig na awitin ng mga gaya ni Hajji Alejandro, na tinaguriang "kilabot ng mga kolehiyala," o ang medyo pilyong tipo ng mga liriko na pinasikat ng gaya ni Rico J. Puno. Ganito rin sa iba pang kategorya ng OPM. Ito man ay ang nakakaindak na indayog ng Manila Sound o ang alternatibong musika ng mga banda gaya ng Juan de la Cruz Band, kapos rin ang espasyo para sa mas malawak na pag-unawa sa usapin ng kasarian kapag sinuri ang mga ideyang inilalako sa kanilang mga kanta. Dagdag pa rito, ito ang panahong sumikat ang grupong Hagibis na naglako ng imahen at mga awiting hindi lang basta heteronormative, kundi brusko at sexist-isang kakatwang kaganapan kapag inisip na ang grupo dapat ang pantapat ng OPM sa Village People (Gabrillo, 2018b). 
Kaya bagama't hindi mapasusubalian ang musika ng protesta na iniluwal ng malawak na kilusang kontra-Marcos (Castro, 2011; Navarro, 2014), masasabing walang masyadong pagbabago sa OPM sa pagitan ng panahon ni Marcos at sa pagtatapos ng diktadurya sa maagang bahagi ng dekada '90. Sa halip, pagpapatuloy ang nangyari (Balance, 2016): napalitan si Hajji Alejandro ng mas batang Ariel Rivera, samantalang nanatili ang brusko at pilyong imahen ng pagkalalaki (at tila naging mas bulgar) na narinig sa mga awiting rap ng mga gaya ni Andrew E. at Legit Misfitz.

\section{Eraserheads bilang Tradisyonal na Banda}

Sa kontekstong inilatag sa itaas umusbong at sumikat ang Eraserheads. Binuo ang banda nina Ely Buendia (bokalista, gitarista), Raimund Marasigan (drummer), Buddy Zabala (bahista), at Marcus Adoro (gitarista), pawang magkakaibigan sa kolehiyo sa Unibersidad ng Pilipinas (UP) sa Diliman sa huling bahagi ng dekada ' 80 . Naging matunog ang kanilang pangalan dahil sa pagtugtog nila sa UP Fair at kalaunan sa mga sikat na bar, gaya ng Club Dredd sa Quezon City (Garcia, 2006). Nagsimula ang pagsikat ng banda noong 1993 nang sunod-sunod na naging "radio hits" ang mga kantang lumabas sa kanilang unang album sa ilalim ng isang prominenteng record label (BMG Records Pilipinas), ang Ultraelectromagneticpop! na naabot ang estadong double platinum (90,000 kopya) para sa dami ng nabentang kopya (Valle, 2000). Hinigitan pa nila ito noong 1994 sa Circus, ang ikalawang album (Valle, 2000). Naabot ng banda ang rurok ng tagumpay sa ikatlong album na Cutterpillow noong 1995. Sa bilang na 320,000 kopyang naibenta, na katumbas ng walong beses na platinum, ang Cutterpillow ang pinakamatagumpay na proyekto ng Eraserheads (Valle, 2000).

Malakas ang hatak ng mga awitin ng Eraserheads dahil sa mga liriko nitong pumapaksa sa iba't ibang aspekto ng pang-araw-araw na buhay ng isang karaniwang Filipino, na kadalasan pa'y gumagamit ng Taglish (Balance, 2016). Ayon sa isang tagahanga:

The band's brilliance lay in their deft use of language, notably through Ely's particular brand of wordplay and wit. Perhaps their most singular achievement was to make Tagalog fashionable, but not in the elegant, mellifluous manner of Ka Freddie nor Asin. The Eraserheads gave my generation a lingo. They tanned and dyed the language to make it supple; they made Tagalog wearable. They knew instinctively that the lingua franca held an unspoken appeal and that it was time to unleash it. (Maneja, 2006, p. 34) 
Ito marahil ang dahilan sa likod ng biglaang paghina ng dami ng benta ng ikaapat na album ng banda, ang Fruitcake (1996). Inilabas ng banda ang Fruitcake bilang soundtrack ng isang malapantasyang librong pambata na isinulat rin ng banda at may kaparehong pamagat (Eraserheads, 1997). Kapuwa nasa wikang Ingles ang libro at lahat ng kanta sa album. Bagama't sinubukan ng banda na bumalik sa nauna nitong tunog at estetika sa ikalimang album na Sticker Happy (1998), hindi na nito nabawi ang naunang nibel ng popularidad. Kalaunan, tinahak nito ang eksperimental na landas sa mga huling album nito: Natin '99 (1999) at Carbon Stereoxide (2001), na kapuwa may malakas na impluwensiyang electronica. Mismong ang mga kasapi ng banda ang umamin na hindi kasimpopular ang mga ito gaya ng mga naunang labas (Valle, 2000). Sa puntong ito, malinaw sa kanila na nagiba na ang kanilang panlasa at oryentasyon mula sa kanilang mga unang taon ng kasikatan. Ani Marasigan, "Our music is just the same, still pop, but we're trying to evolve. We've grown older. We no longer write about homework or cramming because we're not in school anymore" (Valle, 2000). Gayumpaman, hindi maitatatwa ang bigat ng impluwensiya ng Eraserheads: hindi lang nito hinawan ang landas para sa iba pang mga sikat na bandang gumawa rin ng kani-kanilang pangalan sa industriya (hal., Rivermaya, Yano, Color It Red), hinubog din ng banda ang himig ng isang buong henerasyon ng kabataang Filipino.

Bilang tagabandera ng OPM sa dekada '90, nakaangkla ang popularidad ng Eraserheads sa mga kanta nitong halos walang bahid ng politika. Mga kuwento ng pag-ibig sa "Ligaya," "Sembreak," at pagkasawi naman sa "Pare Ko," "Kailan," at "Ang Huling El Bimbo." Indibidwal na kalayaan sa "Alapaap," at "Overdrive." "With a Smile" at "Minsan" bilang awit ng pagkakaibigan. Pawang mga temang madaling sakyan at agad naisapuso ng isang henerasyon ng kabataang Filipino na wala nang direktang kamulatan sa diktadurya ng nakalipas (cf. Claudio, 2012).

Sa usapin ng kasarian, makikita ang tradisyonal na nosyon ng romantikong pag-ibig sa mga pinakasikat nilang kanta. Katunayan, pawang romantikong pag-ibig na heteroseksuwal ang paksa ng lahat ng mga awiting sumikat sa Ultraelectromagneticpop!: i.e., "Ligaya," “Toyang," "Pare Ko," at "Shirley." Maaari pa ngang gawan ng argumento na may bakas ng kontrababaeng pagtingin ang "Shirley" dahil sa animo'y paglalarawan sa dalagang paksa ng kanta bilang mababaw at materyalistiko:

In love na naman si Shirley sa binatang maganda ang kotse.

Sila'y nag-date sa may Antipolo kagabi.

Lagi na siyang naka-dress ng eskwela. 
Nakaayos palagi ang buhok niya.

Lumulutang sa ulap 'pag naglalakad sa kalye. (Buendia \& Marasigan, 1993, 0:20-0:50)

Samantala, "Pare Ko" ang naglagay sa Eraserheads sa posisyon nito bilang bandang hindi lang popular kundi kontrobersiyal. Dulot ito ng prominenteng paggamit ng alimura sa isang linya ng kanta, ang pinakapaboritong mura ng mga Filipino ika nga, at sa mismong koro pa. Subalit bukod sa paggamit ng maaanghang na salita, pumatok ang "Pare Ko" dahil sa estilo ng liriko nito na tila ginagaya ang pinagdadaanan ng persona: Isang binatang nakikipaginuman sa kaniyang matalik na kaibigang lalaki (pare) upang maglabas ng sama ng loob tungkol sa pag-ibig. Malinaw ang heteronormative na dulog ng awitin. Sa katunayan nga, isang pelikula ang inilabas noong 1994 ng Star Cinema na ginamit din ang pamagat ng awit. Tampok sa pelikula ang mga pinakapopular na binatang artista noon: sina Mark Anthony Fernandez, Jomari Yllana, at Jao Mapa. Umiinog ang kuwento sa pag-usbong at pagkasira ng kanilang pagkakaibigan dahil sa pagiging magkaribal ng mga karakter nina Yllana at Mapa para sa isang dalagang ginampanan naman ni Claudine Barretto (Reyes \& Reyes, 1995). Dagdag pa, lubhang salat ang karakterisasyon sa bidang babae, na para bang ang tanging papel sa buong kuwento ay ang maging obheto ng nagbabanggaang macho ego ng mga tauhang ginampanan nina Yllana at Mapa.

Nagpatuloy ang ganitong tema ng heteronormativity sa mga sumikat na kanta sa Circus at Cutterpillow. Halimbawa nito ang "Sembreak" sa ikalawang album at "Ang Huling El Bimbo" mula sa Cutterpillow, ang maituturing na pinakamatagumpay nilang awitin. Nanalo pa nga sila sa MTV Video Music Awards para rito at tinanggap ang kanilang parangal sa New York City (Pastor, 2015). Pero kasabay din ng mga kantang ito ang iba pang awitin na hindi lang sumasandig sa tradisyonal na pananaw sa kasarian, kundi naghahatid ng mga mensaheng sexist, gaya ng kantang "Magasin." Kasama ang "Magasin" sa Circus (Buendia, 1994b) at naging prominente dahil sa kakaiba nitong paksa: ang pagkatuklas ng lalaking persona na ang isang dating kakilalang babae ay isa nang modelo para sa isang malaswang publikasyon. Naaalala pa nga ng persona kung paano niya linait ang itsura ng babae: "Di ko inakalang sisikat ka./ Tinawanan pa kita/ Tinawag mo akong walang hiya./ Medyo pangit ka pa noon/ ngunit ngayon" (Buendia, 1994b, 0:36-0:49). Subalit dahil gumanda na ang babae, nagbago ang pananaw ng persona at gusto nang magkaroon ng perang "Pambili sa mukha [niyang] maganda" (Buendia, 1994b, 1:08-1:16). Nagwakas ang kanta sa pangangarap ng persona, "Sana sa susunod na issue/ Ay centerfold ka na" (Buendia, 1994b, 3:34-3:42). Nasundan pa ang ganitong komodipikasyon 
ng seksuwalidad sa awiting "Superproxy" mula sa Cutterpillow (Buendia and Magalona, 1995). Bagama't hindi lantad ang kasarian ng persona, mahihinuha ang makalalaking suheto ng kanta batay sa agad-agarang pagpapaubaya sa makamundong pagnanasa gamit ang teknolohiya, lalo na sa mga taong nakatali sa isang pangmatagalang relasyon pero may sapat na pera sa "bulsa para guminhawa":

Napapagod ka na ba sa kayayakap sa asawa mo?

Ito(/ako) ang kailangan mo.

I-dial lang ang telepono.

Hindi na dapat maghirap.

Sa iisang iglap ang buhay ay sasarap.

Wag nang mag-atubili kumuha na ng

Superproxy. (Buendia and Magalona, 1995, 0:55-1:36)

\section{"I Go for Flower Power"}

Subalit para sa sanaysay na ito, hindi ang mga sikat na kanta ng Eraserheads ang pagtutuunan, kundi yaong mga awitin na hindi naging bahagi ng regular na playlist sa radyo at hindi pamilyar sa karaniwang Filipino, maliban na lang sa mga masugid na tagasunod ng banda. Sa mga awiting ito, sa aking palagay, nagkukubli ang isang progresibong pananaw sa usapin ng kasarian, partikular na sa isyu ng pagkalalaki.

Sa Ultraelectromagneticpop!, ikaapat na kanta ang "Shake Yer Head." Tungkol ito sa mga internal na tunggalian ng persona habang sinasalag ang bulag na pagsunod sa mga tradisyonal na pagpapahalaga:

I grew up, in a town

where everybody tried to push me around.

The girls were alright,

but the guys were tough;

they're always buggin' me with macho stuff.

Well, I ain't no stupid fighter.

I go for flower power.

I've been running every race just to save my face, yeh.

(Buendia, 1993, 1:00-1:28)

Matagal nang may tradisyon ng pagsalungat o pagkuwestiyon sa awtoridad, na makikita sa simula pa lang ng pag-usbong ng OPM noong dekada '70. Naitanghal ito sa mga awiting “Titser's Enemi No. 1” ng Juan de la Cruz Band at "Estudyante Blues" ni Freddie Aguilar (Maceda, 2007). Inilalarawan din sa mga nasabing kanta ang arketipo ng tigasing lalaki: 
mapusok, hindi umiiwas sa gulo, at lumalaban sa awtoridad ng magulang at paaralan. Sa isang banda, tila kaugnay ito ng diwang kontra-gahum noong panahon ng diktadurya, subalit malinaw na limitadong tipo ito ng rebelyong indibidwalistiko. Isang lalaking rebeldeng sa hulma ni James Dean, at hindi ni Che Guevarra.

Kapag pinalalim ang pagsusuri, masasabi pa ngang ang rebelyong itinutulak sa "Shake Yer Head" ay hindi lang basta pagtuligsa sa awtoridad, kundi sa mismong dominanteng pag-unawa sa pagkalalaki na nakapako sa pamantayang heteronormative. Linalabanan ng persona hindi lang ang kaniyang magulang o ang maangas na siga kundi ang buong ideya na ang lalaki ay dapat mapusok at hindi umiiwas sa gulo. Ang turing ng persona sa ganitong pagpapakahulugan sa pagkalalaki ay "dog food" na sapilitang isinusubo sa lahat, kung kaya't kinakailangang bumalikwas- "macho stuff" na dapat itakwil. Kompara sa malarebeldeng tono ng Juan de la Cruz at ni Aguilar, tila mas malapit pa nga ang Eraserheads sa diwa ng pandaigdigang aktibismo na nasimulan ng dekada '60 at binandera sa katagang "flower power" na pinasikat ni Allen Ginsberg, sampu ng kilusang hippie at kontragiyera sa Kanluran (Wild, 1969, 67). Sa puntong ito, nararapat ring banggitin ang impluwensiya sa Eraserheads ng kontra-kulturang kilusan ng dekada '60. Sa Ultraelectromagneticpop! maririnig ang mga alingawngaw ng kanta ni Natalie Cole, lalo na sa kantang “Toyang," masasagap ang pahiwatig ng paggamit ng marijuana sa album filler na "Ganjazz," at makikita sa disenyong pabalat ang pagsuot ng mga miyembro ng banda ng sapatos na Chuck Taylor. Prominente din sa disenyo ng pabalat ang larawan ng sunflower: tila literal na pagpapatampok sa flower power. Maaaring suriin ito bilang mimicry ng sinakop sa kultura ng mananakop, subalit tila padaskol-daskol ang tumalon sa ganitong kongklusyon (Hedman at Sidel, 2000). Maling akala, sa ganang akin, lalo na kung isaalang-alang ang nilalaman ng iba pang kanta ng Eraserheads.

Isa pang awitin sa Ultraelectromagneticpop! ang "Maling Akala." Sa unang dinig, maihahanay ito sa mga kanta ng Eraserheads na may himig na madaling sabayan at titik na madaling sakyan. Kuwentong katatawanan na umiikot sa mga "butas" at "gusot" na resulta ng maling akala sa pangaraw-araw na buhay, madaling malampasan ang pagbabaligtad na ginawa ng kanta para ilantad ang duwag na lalaking persona, na humantong pa nga sa kaniyang pagiging ander-de-saya, o lalaking takot sa kaniyang babaeng may-bahay:

Nung ako'y musmos pa lamang ay takot sa multo.

Nung ako'y naging binata, sa erpat ng syota ko.

Ngayon ay may asawa. 
Meron nang pamilya.

Wala namang multo ngunit takot sa asawa ko. (Buendia at Marasigan, 1993, 0:34-0:54)

Bagama't may malinaw nang pagkuwestiyon sa tradisyonal na nosyon ng pagkalalaki sa unang album ng Eraserheads, napalalim ang pagtalakay nila sa paksang ito sa ikalawang album, Circus. Sa kantang "Hey, Jay," hindi na lang hinahamon ang heteronormativity, lantaran nang inihahapag ang homoseksuwalidad bilang isyung dapat harapin ng lipunan. Sa unang dalawang saknong, inilatag ang paghihirap ni Jay, ang kaibigang kinakausap ng persona:

Hey, Jay, nabugbog ka na naman daw kahapon.

Hey, Jay, ba't kasi pumunta ka pa sa lugar na 'yon?

Alam mo namang galit sila sa tulad mo.

Alam mo namang 'di ka nila maiintindihan.

Hey, just look to the left, and just look to the right.

Makikita mo naman na hindi pantay-pantay. (Buendia, 1994a, 0:24-1:00)

Kompara sa palabirong tono ng "Shake Yer Head" at "Maling Akala," seryoso ang dulog ng persona habang animo'y ginagamot ang pisikal at emosyonal na sugat ni Jay sa pamamagitan ng pag-uulit ng kataga ng pagasa sa koro: "Hey Jay, everything's gonna be okay." Sa ikalawang stanza naman, naging paghahambing ito sa pagitan ng dalawang magkatunggaling lalaki: si Jay at ang kaniyang ama:

Hey, Jay, nag-away na naman kayo ng tatay mo.

Hey, Jay, wala raw siyang anak na tulad mo.

Alam niya namang wala kang kasalanan.

Alam niya namang ipinanganak ka nang ganyan. (Buendia, 1994a, 1:12-1:39)

Magtatapos ang kanta sa pagtatanong ng persona sa mas malawak na lipunan sa kung paano nito tratuhin yaong mga naiba, at ang paggigiit na tao pa rin tulad ng lahat ang mga gaya ni Jay.

Bakit ba sila ganyan?

Puno ng galit ang isipan.

Ba't ba tayo ganito?

Walang galang sa kapwa

ta-o, ta-o, ta-o. 
Hey, Jay, sa'n ka na ngayon pupunta?

Hey, Jay, kailangan lang ay magtiis ka.

Alam mo namang may iba ka pang magagawa.

Alam mo namang ang mahalaga ay nabubuhay ka.

Jay, whatever you are, and wherever you go,

isip-isipin mo na lang na may nagmamahal sa 'yo.

Now he-he-he-hey, Jay.

Be happy and be gay. (Buendia, 1994a, 2:07-3:23)

Nagtatapos ang kanta sa tila konserbatibong resolusyon ng persona para sa suliranin ng kaibigan: kailangang magtiis ni Jay dahil ang mahalaga ay nabubuhay siya. Maituturing man itong kakapusan sa progresibong politika dahil sa tugon na animo'y "bahala na"-magkantahan na lang tayo (Be happy and be gay!) sa kabila ng estruktural na pang-aapimahirap ding ipagwalang-bahala ang halaga ng kanta sa mas malawak na konteksto ng lipunang Filipino. Sa sitwasyon ng dekada '90 na kulang ng maayos na representasyong LGBTQ sa kulturang popular (Navarro, 2015) samantalang lipos ito sa atrasadong karakterisasyong pinasikat ng mga gaya ni Roderick Paulate, biyaya ang isang kantang gaya ng "Hey Jay" na mula sa isang bandang kasimpopular ng Eraserheads. Ayon nga sa batikang mamamahayag ng OPM na si Eric Caruncho (1996), ang "Hey Jay," na binansagan niyang pinakamahusay na kantang lumabas noong 1994, ang tanging kantang alam niya na may positibong pagtingin sa mga bakla. Hindi man ito nagkaroon ng popularidad gaya ng maraming awitin sa Circus, kongkreto ang talab nito sa mga masugid na tagasubaybay ng banda. Ganito ang lumalabas sa pagbabalik-tanaw ni Chong Ardivilla, isang fan na nagambag sa isang antolohiya ng mga sanaysay tungkol sa Eraserheads noong 2006. Pinamagatang “'Hey, Jay,' Don't Go Away or, How the Eraserheads Took a Sad Song and Made It Happy and Gay," binalikan ng sanaysay ni Ardivilla (2006) ang epekto, aktuwal at potensiyal, sa kaniya ng kanta na tinawag niyang "defiant statement":

1995 was also the year I decided to break free and "out" myself-at least to my friends. I was afraid of disappointing my parents and fearful of being relegated to the margins of society. If "Hey Jay" were to have filled the airwaves then, what a difference it would have made to people like me. And there were a lot of us wrangling with the ugly reality of being "not normal."

But the song played only in the private confines of rooms and Walkmans. Perhaps, society was not ready for that 
song. Perhaps, the Eraserheads were ahead of their time. After all, it was also the same period when the popularity of Roderick Paulate's faggoty caricature of the bakla had reached its apogee. (p. 24)

Gaya ng "Shake Yer Head," maliit na porsiyento lamang ng tagapakinig ng Eraserheads ang pamilyar sa "Hey Jay" kapag ikinompara sa mga mas sikat na kanta. Gayon na lamang ang pithaya ni Ardivilla (2006) para sa isang alternatibong sitwasyong nasa kamalayang "mainstream" ang kantang may simpatya para sa isang bakla. Nakalulungkot lang na hindi ito ang realidad; bagkus, ang mga mismong banda at mang-aawit na nahahanay kasama ng Eraserheads sa kategoryang alternative/rock-and-roll ang kakikitahan ng ugaling kontra-LGBTQ:

Ironically, rock and roll has never been kind to baklas either, in spite of the assurances sung by one of the country's more influential ever...

Homosexuals are still quietly reviled by the movers and shakers of rock \& roll in Manila. I know someone who is gay who fronted a rock \& roll band. One day, his band staged a coup and ousted him. One even suggested that their gay member "tone down" if he wanted to be part of the band because they didn't want to turn off the all-too-powerful music executive. The band mates wanted to live the illusion of the rock \& roll lifestyle, with screaming groupies and corporate-backed angst-ridden lyrics. The gay guy was trumped by The Normalcy Machinery. (Ardivilla, 2006, p. 25)

Kung pagbabatayan ang kuwento ni Ardivilla (2006) tungkol sa bandang hindi niya pinangalanan, kataka-taka pa ba kung bakit hindi pinili ang "Hey Jay" at "Shake Yer Head" ng BMG para patugtugin sa radyo? Tanggapin bilang produktong sining ng banda subalit ikubli mula sa madla para hindi na pag-usapan-karanasang kawangis ng pinagdaanan nina Ardivilla at Jay.

\section{Sa Daigdig ng mga NPA, Torpe, at Diwata ng Prutas}

Sa gitna ng tagumpay ng Cutterpillow hindi nawala sa mga katha ng Eraserheads ang mga di-tradisyonal na pag-unawa sa pagkalalaki. Sa katunayan, tila lalo pa itong napatampok at napalalim, partikular na sa dalawang kanta sa nasabing album: ang "Yoko" at "Torpedo." 
May bagong anggulong sinuong ang kritisismo ng banda sa "Yoko" dahil lantaran nitong tinuligsa ang estado sa paghulma ng militarisadong kamalayan sa hanay ng kabataan. Isang sistematikong puna kontra sa Citizens' Military Training (CMT) ang buod nito:

Nasayang ang maghapon, ano ang napala?

Basura sa utak, sunburn sa batok at noo.

Nagmamartsang parang gago sa ilalim ng araw.

Baril na kahoy pinaikot-ikot parang langaw.

Paano irerespeto opiser na bobo?

Puro "demit-kadit," natutulili na ako.

Parang tooth decay, patakarang walang silbi.

Minsan gusto ko nang sumali ng NPA

and blow them shit away.

Tigilan na ang raket, raket ng gobyerno.

'Di ko na kailangan ang pang-aabuso n'yo.

Ginagawa kang papet, papet ng army.

Yoko na, yoko na, yoko na, yoko na

Yoko nang mag-CMT! (Marasigan, 1995, 0:01-0:54)

Nagmimistulang makitid ang pagtuligsa sa lipunan na narinig sa "Shake Yer Head" kapag ikinompara sa bigat ng pagpuna sa "Yoko." Sa halip na isang abstraktong paglalarawan sa machismo, isang kongkretong manipestasyon ng militarisadong pagkalalaki ang target ng "Yoko." Binabaklas sa bawat taludtod ang isang institusyon ng pamahalaan na pinagdaraanan noon ng lahat ng mag-aaral sa kolehiyo sa Pilipinas. Sa halip na itanghal ang kadete bilang personipikasyon ng disiplinadong karahasan sa katawang lalaki, kinukutya ng mang-aawit hindi lang ang kahangalan ng kaniyang opiser, kundi ang kabulukan ng estado. Mas masahol pa sa pagiging sayang sa oras, isang malaking raket ng mapang-abusong gobyerno ang CMT. Higit pang magkakalaman ang kritika ng "Yoko" kapag isinakonteksto sa maagang bahagi ng dekada '90, kung kailan pangulo ng bansa si Fidel Ramos, na dating hepe ng konstabularyo at vice-chief of staff ng Sandatahang Lakas ni Marcos, kapagdaka'y naging bayani ng EDSA People Power at pangkalahatang pinuno ng Sandatahang Lakas ng administrasyong Corazon Aquino. Ito rin ang dekada kung kailan nanalong senador ang kontra-komunista at kanangkamay ni Marcos na si Juan Ponce Enrile at si Gregorio Honasan, na tangan ang imahen bilang repormista-populistang rebeldeng-sundalo. At nanalo sina Enrile at Honasan sa kabila ng pagtatambalan ng dalawa sa mga kudeta laban kay Aquino. Ito rin ang dekadang lugmok ang Partido Komunista ng Pilipinas at ang armadong sangay nitong New People's Army (NPA) dulot 
ng mga internal at panlabas na krisis, samantalang nag-aagawan sa puwesto sa gobyerno ang mga dating opisyales ng kasundaluhan (McCoy, 1999). Sa kontekstong ito mapapalutang ang sidhi ng galit ng persona na nakuhang ibulalas na: "Minsan gusto ko nang sumali ng NPA/ and blow them shit away." Ano na lang kaya ang dagdag na lalim ng paghahambing na ito sakaling napaaga nang isang dekada ang unang pag-iisang-dibdib sa pagitan ng dalawang baklang kadre ng NPA na nangyari noong 2005 (Madula, 2016)?

Bagama't matalim ang batikos ng "Yoko," tulad ng mga sinuring kanta sa itaas, limitado ang impluwensiya nito dahil hindi ito kasama sa mga kanta ng Cutterpillow na regular na pinatutugtog sa radyo. Dito ngayon papasok ang kakaibang talab ng “Torpedo." Pareho mang kritikal sa dominanteng imahen ng heteroseksuwal na pagkalalaki, tila nasa magkabilang dulo ng ispektrum ang "Yoko" at "Torpedo" pagdating sa tema at tunog. Kung ngitngit sa gobyerno ang namamayaning damdamin sa unang kanta, marubdob na awa sa sarili naman ang para sa ikalawa. Kung opensiba kontra estado ang atake ng nauna, pagsuko ng isang torpeng ni hindi nga makapanligaw ang dalamhati ng ikalawa. Tila pang-uuyam na isang pampasabog sa digmaan ang resulta ng paglalaro ng banda sa salitang torpe na ginamit nilang pamagat. $\mathrm{O}$ nakaasinta ba ang pasabog sa kumpiyansa ng abang lalaking persona?

Pasensya na

kung ako ay

'di nagsasalita.

Hindi ko kayang sabihin

ang aking

nadarama.

Huwag mo na akong pilitin.

Ako ay walang lakas ng loob

para tumanggi.

Walang dapat ipagtaka.

Ako ay ipinanganak na torpe,

sa ayaw at hindi.

Pasensya na

kung ikaw ay naiinis.

Ayoko na sanang pag-usapan pa.

Kung gusto mo ay

manood ka na lang ng sine.

'Di ba Huwebes ngayon, 
baka may bago nang palabas?

Huwag mo na akong pilitin.

Ako ay walang lakas ng loob

para tumanggi.

Walang dapat ipagtaka.

Ako ay ipinanganak na torpe

diyan sa tabi-tabi.

Pasensya na

kung ako ay naiiyak.

Mababaw lang talaga ang luha ko.

'Di ko mapigil

ang aking damdamin.

Puwede bang umalis ka na,

tumutunog na ang beeper mo? (Buendia, 1995, 0:01-2:56)

Halos uutal-utal ang pagkanta ni Buendia na pinuputol sa alanganing hati ang mga taludtod. At animo'y pira-pirasong pinupulot ng persona ang kaniyang nabasag na ego sa pagtatapos ng kanta. Kapag inihambing sa "Shake Yer Head" at "Yoko," lubhang mas malalim ang artikulasyon nito ng alternatibong pag-unawa sa kasarian sapagkat hindi lang basta nito itinatanghal ang mga gawi na taliwas sa ideyal na pagkalalaki (pagiging torpe; kawalan ng lakas ng loob; pagtaboy at-iqué horror!-pag-iyak sa harap ng napupusuang babae), malinaw sa persona na wala siyang balak makipagtuos. Samantalang may pagtuligsang nauulinigan sa "Shake Yer Head" at "Yoko," lumbay, pag-amin sa kabiguan, at paulit-ulit na paghingi ng pasensiya ang "resolusyon" sa pinagdadaanan ng torpe. Wala itong balak sumali sa "pataasan ng ihi" o anumang "larong panlalaki, larong macho, larong agawan ng kapangyarihan o poder o gahum" (Cruz, 2002, p. 114). Kay lakas ng mensaheng hatid ng isang lalaking tumatanggi mismo sa maka-lalaking ideya ng lakas. Pero ang pinakakritikal na punto sa lahat: ang "Torpedo" ay nagkaroon ng masiglang airplay sa radyo at naging popular.

Bagama't mukhang pinakamabisa ang “Torpedo" sa lahat ng mga kanta ng Eraserheads na natalakay sa puntong ito pagdating sa pagpapatampok ng isang alternatibong pag-unawa sa pagkalalaki, maaari ring igiit na hindi kanta ang naglahad ng pinakasiksik na artikulasyon ng banda hinggil sa isyu ng kasarian, kundi sa isang kuwentong pambata. Isang taon matapos ang paglabas ng Cutterpillow, isinapubliko ng banda ang kasunod na proyekto: Fruitcake, isang album na may temang pampasko. Dagdag pa rito, inianunsiyo rin ng banda na maglalathala sila ng isang librong pambata na may kuwentong batay sa mga kanta sa album na Fruitcake. Samakatwid, 
maituturing na soundtrack ang ikaapat na album ng banda para sa librong may pamagat ding Fruitcake. Pinamatnugutan ni Jessica Zafra, inilimbag ang libro ng Anvil noong 1997. Sa aking palagay, sa parehong album at librong Fruitcake pinakalantad ang henyo ng Eraserheads pagdating sa pagbasag sa heteronormative na pag-unawa sa pagkalalaki.

May pagbasag na agad na makikita sa pangkalahatang konseptong pumapalibot sa Fruitcake bilang isang proyektong album-at-libro. Una, ang mismong paggawa ng isang album na pampasko ay isang bagay na hindi iniuugnay sa mga alternatibong banda, na kadalasan pa nga'y naikakahon bilang mga di-huwarang indibidwal na Satanista o di kaya ay durugista (Gabrillo, 2018a). Madalas ring iniuugnay ang ganitong konsepto ng album sa mga babaeng mang-aawit gaya ni Nora Aunor o ng mga higit na malumanay na lalaking balladeer gaya ni Jose Mari Chan. Idagdag pa rito ang pagsabak ng banda sa paglathala ng librong pambata. Tila taliwas sa imahen ng rakistang lalaki ang mahanay sa linya ng pagsasalaysay ng mga kuwentong-bagama't may mga pailalim na temang pangmatandanakatuon sa mga batang nag-aaral pa sa elementarya, lalo pa't isang batang babae ang bida.

Umiikot kay Frannie Wei ang kuwento ng librong Fruitcake (Eraserheads, 1997). Naglayas si Frannie at napadpad sa Fruitcake Heights, ang pandaigdigang sentro ng paghahanda at pagluluto ng pamaskong pagkain na fruitcake at kung saan nakilala niya ang Carole Kings, isang bandang binubuo ng apat na miyembro at klarong kumakatawan sa mga miyembro ng Eraserheads. Kung hindi masaya si Frannie sa tahanan niya, dismayado naman ang Carole Kings dahil paulit-ulit ang mga kantang pamasko na pilit ipinatugtog sa kanila ng mga Fruitcakeans, ang taguri sa mga residente ng Fruticake Heights. Humantong ang monotoniyang ito sa pagkalat ng isang sakit sa komunidad na tinawag na "monovirus."

Kung ikokompara sa Ultraelectromagneticpop!, Circus, at Cutterpillow, ibang-iba ang tunog at pangkalahatang estetika ng Fruitcake. Sa kabuuan ng libro at ng album, makikita ang pagsusumubok ng Eraserheads na maghawan ng bagong landas pagdating sa mga tema at paksa, palayo nang palayo sa nakagisnan ng publiko sa unang tatlong album, gaya na lang ng pagnanais ng Carole Kings na makaalis sa Fruitcake Heights. Subalit sa halip na ilahad sa porma ng mapusok at marahas na pagbalikwas, idinaan ng Fruitcake ang pagwasak ng tradisyon sa mapanuya at mapaglarong mga paraan. Paboritong lugar ni Santa Claus ang Fruitcake Heights, isang lugar kung saan walang niyebe, kung kaya't nagtitiyaga ang mga bata na maglaro sa ulan ng pira-pirasong styrofoam, o styrosnow, na nagmumula sa tsimeneya ng isang pabrika. Naging gamot sa monovirus ng mga Fruitcakeans ang pagpapakain sa kanila ni Frannie ng bibingka at puto bumbong. Ang 
pinakamatalinong pantas ng Fruitcake Heights ay nagngangalang Bobo the Wiseman na naisahan ni Frannie nang dalawang beses: una, sa pamamagitan ng pagbibigay ng bagong impormasyon sa tulong ng isang simpleng diyaryo; ikalawa, sa pagkakatalo ni Bobo sa batang bida sa larong Trip to Jerusalem.

Hindi limitado ang dekonstruksiyon ng Fruitcake sa tila postkolonyal na pagbaklas at muling pagbuo sa Kanluraning tradisyon ng Pasko, sapagkat maging ang usapin ng kasarian ay hindi pinalampas ng mga may-akda. Isa ang Fruit Fairy sa mga prominenteng personalidad sa Fruitcake Heights na nakilala ni Frannie. Agad na tatambad ang isyu ng kasarian sa kanilang unang pagtatagpo: "There was a puff of smoke in front of Frannie and a figure appeared, coughing. When the smoke cleared, Frannie was surprised to see not a young girl, but a grown man. Wearing ballet shoes and a tutu. 'Voila,' said the man. 'It is I, le Fruit Fairy.' He waved his wand and batted his eyelashes" (Eraserheads, 1997, p. 63). May katambal na kanta sa album ang engkuwentrong ito sa libro, na pinamagatan ding "Fruit Fairy":

Magic in the vine, sugar when I cook.

Shake and bake the flavor, come and take a look.

Nuts, nuts, nuts everywhere.

Berries and wine so divine they grow in my hair.

It ain't easy being a fruit fairy.

Fruits are flights of fancy.

Just think fruitcakes ain't no fruitcakes without me.

Here, take this wand, wave it in the air.

Surprise, surprise, that really is my spatula.

All day long I tend the garden, talk to the leaves, and dig the sun. (Mangulabnan, Marasigan, \& Zabala, 1996, 0:12-1:07)

Bagama't katatawanan kaysa aral ang nais ibahagi ng Fruitcake sa mambabasa, hindi pakay ng libro na gawing punchline ang karakter na Fruit Fairy. Matapos siyang ipakilala sa nabanggit na tagpo, hindi na bibigyangpansin muli ang alanganing paglalarawan sa kaniyang kasarian-malayo sa kadalasang pagtrato ng kulturang popular sa Pilipinas sa mga tauhang hindi pumapasok sa mga kategoryang heteronormative.

Kuwento man ito ng pagpiglas mula sa tradisyon, nagtatapos ang Fruitcake sa pagbabalik ng mga pangunahing tauhan sa piling ng nakasanayan: ang Carole Kings sa Fruitcake Heights at si Frannie sa kaniyang ina. Binigyang kulay ang tagpong ito ng huling kanta ng album, 
na may pamagat na "Christmas Morning." Sa kantang ito, nanay ni Frannie ang persona at inaawitan ang anak na nahihimbing. Mala-oyayi ang komposisyon nito, na ibang-iba kung ihahambing sa rakistang personalidad ng Eraserheads.

Wake up little darling, it's Christmas morning.

The sun is up, it's time to open

all the gifts that you've been given.

You had been dreaming.

Angels are singing.

But now they've gone

and once again it's time to go on with our lives.

Let's hope that the next year will be better than last year and somehow we'd all get by.

And maybe the time will come

when we won't need to pretend to be happy for just a while.

(Buendia, 1996a, 0:25-1:34)

Magwawakas ang himig sa mga taludtod na nagbibigay ng katiyakan sa anak na kakagaling lang sa isang karanasang nagpabago sa kaniyang buhay:

Wake up little darling,

it's Christmas morning.

So rise and shine, let's make the most of happy holidays.

Or maybe I should just let you dream for a while

Longer,

wherever's

a better place than here. (Buendia, 1996a, 2:48-3:34)

Sinong mag-aakalang magmumula sa pinakamatagumpay na rock band ng bansa ang isang kantang nagtatampok ng isang inang ipinaghehele ang kaniyang babaeng anak upang makahimbing nang payapa sa umaga ng Pasko?

\section{Heteronormativity at ang Tulak ng Merkado}

Sa isang banda, hindi dapat ikagulat ang imahen ng Eraserheads na hindi pasok nang husto sa hulma ng lalaking rakista na kakikitahan ng gaspang at lakas. Simula't sapol, ibang-iba ang paraan ng pagkanta ni Buendia kapag inihambing sa mga kasabayan niyang higit na kilala sa "panlalaking" bigat at halos pasigaw na pag-awit na madalas na iniiuugnay sa rock and roll, gaya ng mga bokalistang sina Basti Artadi ng Wolfgang at Glenn Jacinto 
ng Teeth. Kay Buendia, higit ang tinis kaysa tigas pagdating sa timbre. Datapuwa't, hindi ito nangangahulugang walang bahid ng makalalaking heteronormativity ang Eraserheads, gaya ng nailatag sa simula ng sanaysay.

Pero batay sa mga nagtutunggaling perspektiba sa pagkalalaki na maririnig sa kanilang awitin, paano ba dapat ang Eraserheads gamit ang lente ng usaping pangkasarian? Kabalintunaan ba o pagiging ipokrito ang mga ganitong nagbabanggang mensahe? Marahil, hindi sapat ang liriko ng mga kanta bilang tanging batis sa pagsusuri. Kinakailangan ding isaalangalang na, gaya sa iba pang larangan ng kulturang popular, kritikal na salik ang pulso ng merkado para sa paglikha ng sining. At bagama't tapos na noong dekada ' 90 ang diktaduryang Marcos at ang pagbusal nito sa midya, masasabing naging porma rin ng sensura ang dikta ng kapital.

Maliban sa "Torpedo," pawang mga kantang hindi sumikat ang mga nabanggit na awitin ng Eraserheads na nagtatanghal ng alternatibong pananaw ukol sa pagkalalaki. Kabaligtaran nito ang mga kantang sinuri sa simula ng sanaysay na pare-parehong nakalasap ng kasikatan-samakatwid, yaong mga nabigyan ng higit na pansin ng mga punong ehekutibo ng BMG, ang record label ng banda. Hindi dapat kalimutan na sa kabila ng tila pagtuligsa ng Eraserheads sa awtoridad at mga pagpapahalagang tradisyonal, naglalako sila ng kulturang popular na ginagawa para sa kita (Tolentino, 2001) - at sa kasong ito, para sa tubo ng isang dambuhalang transnasyonal na korporasyon. Para sa mga ehekutibo ng BMG, higit sa estetika o potensiyal para sa progresibong politika, ang pagbenta ng mas maraming kopya ng album ang namamayaning layunin sa likod ng kanilang pagpapasiya kung aling mga kanta ang patutugtugin sa radyo, kung alin ang mananatili lang sa album, at kung alin ang hindi katanggap-tanggap.

Bagama't konektado, may malinaw na dibisyon sa pagitan ng estetikong produksiyon at materyal na produksiyon ng musika (Concepcion, 2012). Batay rito, maaaring may progresibong nilalaman ang isang partikular na likhang-sining sa kulturang popular, subalit bilang mga produktong pinakikilos ng kapital, mayroon pa ring mga hanggahan. Mulát na ang banda sa hubad na katotohanang ito noon pa man. Ayon kay Marasigan (sa Caruncho, 1996) sa isang panayam noong 1994: "Hindi sanay ang record companies sa banda na hindi pina-package . . . Sanay sila sa banda na lahat ng miyembro pogi. Kami naman against kami sa norms ng recording industry" (p. 108). Pero pagkalaki-laki man ng tunggalian ng mang-aawit at prodyuser, sa paggawa pa rin ng album ang tuloy. Ayon kay Caruncho (1996):

After being turned down by numerous record companies for not being 'pop' enough (whatever that means), the 
flannel-clad foursome land a recording contract with BMG Pilipinas and begin recording their first album, ultraelectromagneticpop! Record company execs express some misgivings about 'Pare Ko', an otherwise straightforward boy-meets-girl, girl-shafts-boy love ditty, because the lyrics include the key phrases "leche," "tang ina," "nabuburat," etc. As a compromise, the band remixes a sanitized, radio-friendly version with "walanghiya" substituted for "tang ina," "naiinis" for "nabuburat" and so on. (p. 105)

Mapanuksong ituring na "hidden transcripts" (Scott, 1990) ang mga kantang di-sikat ng Eraserheads. Sa ganitong pag-unawa, nakakubli sa ilalim ng hayagang pagtalima ng banda sa lohika ng tubo (na kinakatawan ng kanilang mga sikat subalit heteronormative na kanta) ang teksto ng pakikibaka laban sa mga atrasadong panlipunang estruktura (na kinakatawan ng kanilang mga di-sikat na awiting nagtataguyod ng alternatibong tingin sa kasarian). Sabi nga nila sa kantang "Fruitcake," "There are B-sides to every story/If you decide to have some fun" (Buendia, 1996b, 0:44-0:54). Datapuwa't ang tuksong ito'y marapat layuan; bagkus, mainam na balikan ang paalala ni Craig Lockard (1996):

$[B]$ oth the public and the controlling elites influence the music in a dialectical process; the intentions of the musicians also exert an influence. Hence, music is a double-edged sword-escapist and faddish on the one hand, dynamic and progressive on the other. (p. 153)

Bilang isang komodipikadong sining, may talim at purol ang kulturang popular gaya ng mga kanta ng Eraserheads. Sa lipunang post-EDSA, sa halip na estado ang may kontrol sa kung ano ang pinatutugtog sa radyo, ang dikta ng tubo ang may pinakamalakas na hatak sa andar ng komersiyalisadong sining.

Dagdag pa rito, malinaw na may iba pang nakahihigit sa Eraserheads sa pagpapalitaw ng progresibong politika gamit ang musikang popular bilang behikulo. Nariyan ang The Jerks at Yano, na kapuwa kasabayan din ng Eraserheads at nagkaroon din ng kontrata sa ilalim ng malalaking kompanya (i.e., Star Records at BMG Records), subalit napanatili ang radikal na komentaryo sa kanilang mga komposisyon. The Jerks ang utak sa likod ng awiting maituturing na pinakamatalas na pagsusuri ng lipunang post-EDSA: "Ang Sayaw sa Bubog" (San Juan, 2019). Samantala, kompara sa pagtingin ng "Yoko" sa CMT, higit na komprehensibo ang "Tara” ng Yano sa pagbasag 
nito sa kabuuang sistema ng militarisasyon at ang epekto nito sa taumbayan, na pinabigat pa ng payak subalit tuwirang tono ng pagkakasulat. Malaman ang anggulo ng kasarian sa koro nito sa pagtutumbalik ng militarisadong katawan na tila isip-batang persona na niyayaya ang tagapakinig sa mga larong ikapapahamak ng mga tao sa paligid, mga aktuwal na bata at kanilang mga ina:

Tara, laro tayo ng baril-barilan.

Magkalaban tayo, ratratan ng machine gun.

Tara, laro tayo ng tora-tora.

Magkalaban tayo, hagisan ng granada.

May inang may kargang anak, may batang hubo, umiiyak.

May inang may kargang anak, may batang hubo, umiiyak. (Abay at Gancio, 1997, 0:11$0: 54)$

Kung gayon, pinatutunayan ba ng halimbawa ng Eraserheads ang argumento ni Lockard (1996) na kadalasang may espasyo para sa mga kritikal na pananaw sa kulturang popular, subalit madalas mababaw ang mga ito? Mas mainam sagutin ang tanong na ito sa pamamagitan ng pagtingin sa naging impluwensiya ng banda sa kulturang popular nitong mga dekadang nakalipas.

\section{Toyang sa Panahon ng Tokhang}

Pagmamalabis na ituring ang Eraserheads bilang bandang nagbigay artikulasyon sa politika ng kaniyang panahon. Gayumpaman, bahagi sila ng mas malawak na transisyong kultural na tagatawid ng tinaguriang martial law babies at ng henerasyon ng EDSA. Hindi na dinanas ng kabataan ng dekada '90 ang diktadurya, subalit ang musikang pinakinggan nila ay produkto ng mga binatang namulat ang isipan sa naunang dekada. Matingkad ang ganitong pagtutulay ng dalawang henerasyon sa pamagat ng unang album, na isang alusyon sa palabas na Voltes $V$, isang Hapones na anime na naging kontrobersiyal sa Pilipinas dahil pinutol ng rehimeng Marcos ang pagpapalabas nito sa bansa dahil raw sa karahasang itinuturo nito sa kabataan (Lent, 1998, p. 247). Samakatwid, malaking bahagi ng kamalayan nina Buendia, Marasigan, Adoro, at Zabala, habang lumalaki, ang institusyon ng sensura ng diktadurya sa midyang pangmasa. Subalit may talab pa ba ang banda sa kasalukuyang henerasyon?

Kamakailan, isang musikal na isinulat ni Dingdong Novenario ang itinanghal na may kuwentong batay sa pinagsama-samang ideya mula sa 
ilang piling kanta ng Eraserheads. Pinamagatang Ang Huling El Bimbo ang musikal, halaw mula sa pinakasikat na awitin ng banda. Nagsimula ang unang pagpapalabas, sa direksiyon ni Dexter Santos, noong ika-20 ng Hulyo 2018. Ayon kay Santos: "Their songs make up the soundtrack of our everyday lives, back when we we [sic] fell in love and got hurt for the first time, to the time we were having drinks with our barkada, or having oneon-one emo talks with our best friend" (Perez, 2018). Sa isang banda, ang naratibong nabuo sa pinaghalo-halong kuwentong mula sa mga kanta ng Eraserheads ay siya ring kuwento ng mga nagkamalay noong dekada '90.

Tampok sa dulang Ang Huling El Bimbo (Manalang \& Santos, 2018) ang pagkakaibigan ng apat na tauhan, sina Joy, Hector, AJ, at Emman. At sa paglalahad ng kanilang kuwentong batay sa mga kanta ng Eraserheads, hindi kataka-takang isa sa mga prominenteng tema ang pagkuwestiyon sa konsepto ng pagkalalaki. Sa katunayan, maituturing na pagsasabuhay ng kantang "Hey, Jay," ang karakter ni AJ, na ang pangalan ay malinaw na paglalaro sa pamagat ng kanta. Naging kritikal na bahagi ng internal na tunggalian para kay AJ ang kaniyang pagiging bading, lalo pa't isang heneral ang kaniyang ama at nagpakasal siya sa isang babae. Katunayan, dahil sa kaniyang alanganing relasyon sa asawa, nagkaroon ng matalinghagang pagbabaligtad sa kantang "Kailan" (mula sa album na Circus) partikular sa mga taludtod na "Kailan mo ako hahagkan?/ Matagal na akong naghihintay." Sa orihinal na kanta, ang pangangarap ng persona para yakapin siya ng kaniyang sinisinta ay dulot ng pangungulila sa loob ng piitan ("Nangingig sa seldang maginaw."). At dahil walang malinaw na binabanggit sa liriko, tila nakasanayan ng tagapakinig na isiping lalaki ang persona ng kanta, lalo't isa siyang bilanggo. Subalit sa dulang Ang Huling El Bimbo, ang asawa ni $\mathrm{AJ}$ ang siyang kumakanta at nagsusumamo na "mahagkan" ng kaniyang asawang bading. At ang tugon ni AJ sa dulog ng asawa? Ang pagkanta ng mga linyang "Pasensiya na..." na halaw sa liriko ng "Torpedo." Gayumpaman, dapat ring banggitin ang mga puna sa tila kontra-babaeng mensahe ng dula, lalo na sa naging dulog nito sa panggagahasa kay Joy, na siyang lunsaran ng pangunahing tunggalian sa kuwento. Samantalang naging tuntungan ang alaala ng insidente para sa realisasyon ng tatlong bidang lalaki sa kabila ng kawalan nila ng aksyon matapos masaksihan ang krimen, tila iniwang nakabinbin ang resolusyon para sa mismong biktima (Briñas, 2020).

Mababanaag din ang bisa ng mensahe ng Eraserheads sa mga mangaawit na naimpluwensiyahan nila. Ang bandang Twisted Halo, na kinabibilangan din ng bahistang si Zabala, ay sumikat sa mainstream dala ng kanilang awiting "Brad" (Dancel, 2001), isang malalim na kritisismo sa karahasan at sablay na lohika ng makalalaking kapatiran at fraternity hazing. Samantala, litaw din ang impluwensiya ng Eraserheads sa tanyag 
na rapper na si Gloc-9. Si Gloc-9 mismo ang umamin sa positibong epekto ng banda sa kaniyang musika, at sa katunayan ay nag-ambag ng kanta sa lumabas na tribute album para sa kanila noong 2012 (ABS-CBN Star Music, 2012). Pero maaari pa ngang sabihing nahigitan ni Gloc-9 ang Eraserheads sa pagkuwestiyon sa dominanteng ideya ng pagkalalaki sa kaniyang mga awitin. Halimbawa ang "Magda" (Pollisco, 2013) na kuwento ng isang babaeng nalugmok sa prostitusyon, subalit sa halip na magtapos ang kanta sa mala-bayaning pagsagip sa kaniya ng lalaking persona, na may pangalang Ernesto at kababata ni Magda, pinili ni Gloc-9 na kilalanin ang kakayahan ni Magda na magpasiya para sa sarili at hindi magpatali sa naratibo ng biktimang nawalan ng puri. Samantala, inilalahad sa "Sirena" (Pollisco, 2012) ang mga pinagdaanang pagsubok ng isang bading na nakaranas ng pisikal na pang-aabuso mula sa sariling ama. Kung tila peministang pagbabaligtad ang "Magda" sa makalalaking konsumerismo at titig (gaze) ng "Magsasin," ang "Sirena" naman ay mas palaban (sa paraang progresibo at hindi brusko) na bersiyon ng "Hey, Jay." Batay sa mga kantang ito, at iba pang kawangis na awiting naglalaman ng matalas na pagsusuri ng lipunan, hindi katakatakang tinawag si Gloc-9 ng isang iskolar bilang "organic intellectual" (Mendoza, 2017, n.p.).

Hindi layon ng sanaysay na ito na ituring ang progresibong politika ni Gloc-9 bilang utang niya sa Eraserheads. Gayundin, walang pagnanais ang papel na ito na isantabi ang komersiyalisadong kalikasan ng mga awit ng banda, pati na ang mga malinaw na makalalaking mensahe sa ilang kanta. Bilang isang Filipinong lumaking nakikinig sa musika ng banda, nagsilbing paraan ang akdang ito upang aking kritikal na timbangin ang isang maimpluwensiyang bahaging kabataan ko. Atbatay sa mga nasabi na, sa kabila ng mga puna, malaki pa rin ang potensyal ng Eraserheads sa pagsusulong ng progresibong pulitika pagdating sa usapin ng kasarian. Pinatutunayan nito ang nasabi na ng ibang iskolar tungkol sa mga awiting popular na, gayong may limitasyon, binhi para sa mga ideyang kontra-gahum (cf. Devilles, 2015). Mahalagang pagnilayan ang isyung nakakubli sa mga di-popular na awitin ng Eraserheads, lalo pa't nasa panahon tayo ngayon ng tumitinding krisis sa pagsagka sa toxic masculinity, komodipikasyon ng katawan, at mga sistematikong atake sa mga LGBTQ, at iba pang kahalintulad na suliranin. Kritikal na interbensiyon ang pagbabalik-tanaw sa kulturang popular na iniwan ng banda, ano't anupaman ang kanilang kapintasan, sa yugtong ito ng kasaysayan na lantarang ibinabandera ang populistang machismo bilang ideyal na pagkalalaki upang maisalba diumano ang bayang Filipino. Sa yugtong ito na naghahari-harian ang mga Duterte, Sotto, Tulfo, at Bato, marapat lang pakinggang muli ang natabunang mensahe nina Buendia, Zabala, Marasigan, at Adoro. 


\section{Sanggunian}

Abay, W. M. and E. Gancio. (1997). Tara. On Tara. Musiko Records and BMG Records Pilipinas.

ABS-CBN Star Music. (2012). The Reunion interview: GLOC-9 [Video]. Youtube. https://www.youtube. $\mathrm{com} /$ watch?v=_kl5Dw9vQqu\&t=12s.

Ardivilla, C. (2006). 'Hey, Jay,' don't go away or, how the Eraserheads took a sad song and made it happy and gay. In M. Calimag and J. Garcia (Comp.), Tikman ang langit: An anthology of the Eraserheads (pp. 23-26). Visual Print.

Balance, C.B. (2016). Tropical renditions: Making musical scenes in Filipino America. Durham: Duke University Press.

Briñas, J. R. (2020, 13 May). How "Ang huling el bimbo: The musical" fails women, despite what its supporters say? Medium. Retrieved from https://medium.com/@johannarosebrinas/how-anghuling-el-bimbo-the-musical-fails-women-despite-what-its-supporters-may-say-ea5d943aa819.

Buendia, E. B. (1993). Shake yer head. On Ultraelectromagneticpop! Musiko Records and BMG Records Pilipinas.

Buendia, E. B. (1994a). Hey, Jay. On Circus. Musiko Records and BMG Records Pilipinas.

Buendia, E. B. (1994b). Magasin. On Circus. Musiko Records and BMG Records Pilipinas.

Buendia, E. B. (1996a). Christmas morning. On Fruitcake. Greater East Asia Music and BMG Records Pilipinas.

Buendia, E. B. (1996b). Fruitcake. On Fruitcake. Greater East Asia Music and BMG Records Pilipinas.

Buendia, E. B. and F. D. Magalona. (1995). Superproxy. On Cutterpillow. Musiko Records and BMG Records Pilipinas.

Buendia, E. B. and R. P. Marasigan. (1993). Maling akala. On Ultraelectromagneticpop! Musiko Records and BMG Records Pilipinas.

Buendia, E. B. and R. P. Marasigan. (1993). Shirley. On Ultraelectromagneticpop! Musiko Records and BMG Records Pilipinas.

Cañete, R. R. (2014). Masculinity, media, and their publics in the Philippines. University of the Philippines Press.

Caruncho, E. S. (1996). Punks, poets, poseurs: Reportage on Pinoy rock \& roll. Anvil.

Castro, C. (2011). Musical renderings of the Philippine nation. Oxford University Press.

Claudio, L. (2012, January 1). Were the Eraserheads the end of cultural history? GMA News Online. Retrieved from https://www.gmanetwork.com/news/opinion/content/243261/were-the-eraserheads-theend-of-cultural-history/story/.

Concepcion, D. A. (2012). Embedded tunes: An exploratory sociological study on popular music consumption among selected Filipino workers [Unpublished master's thesis]. Ateneo de Manila University.

Cruz, I. R. (2002). Ang kabastusan ng mga Pilipino. In S.S. Reyes (Ed.), Reading popular culture (pp. 105130). Office of Research and Publications, Ateneo de Manila University.

Dancel, V. (2001). Brad. On Twisted Halo. Guilty Pleasure Records.

Devilles, G. C. (2015). Ang estetika ng reiterasyon sa awiting popular bilang siste ng diskursong kontragahum. In R. B. Tolentino and G. C. Devilles (Eds.), Kritikal na espasyo ng kulturang popular (pp. 106122). Ateneo de Manila University Press.

Eraserheads. (1997). Fruitcake, J. Zafra (Ed.). Anvil. 
Gabrillo, J. (2018a). Rak en rol: The influence of psychedelic culture in Philippine music. Rock Music Studies, 5(3), 257-74.

Gabrillo, J. (2018b). The new Manila Sound: Music and mass culture, 1990s and beyond [Unpublished doctoral dissertation]. University of Cambridge.

Garcia, J. (2006). A dreddful story (with many digressions). In M. Calimag and J. Garcia (Comp.), Tikman ang langit: An anthology of the Eraserheads (pp. 77-83). Visual Print.

Hedman, E. E. and J. T. Sidel. (2000). Philippine politics and society in the twentieth century: Colonial legacies, post-colonial trajectories. Routledge.

Lent, J. A. (1998). Comic art in the Philippines. Philippine Studies, 46(2), 236-48.

Lockard, C. A. (1996). Popular musics and politics in modern Southeast Asia: A comparative analysis. Asian Music, 27(2), 149-99.

Maceda, T. G. (2007). Problematizing the popular: The dynamics of Pinoy pop(ular) music and popular protest music. Inter-Asia Cultural Studies, 8(3), 390-413.

Madula, R. D. (2016). Kuwentong buhay ng isang pambansa-demokratikong rebolusyonaryong bakla sa rehiyon ng Katimugang Mindanao. Humanities Diliman, 13(2), 27-48.

Manalang, C. \& Santos, D. (2018). Ang Huling El Bimbo. By D. Novenario. [Theater production]. Pasay City: Newport Performing Arts Theater.

Maneja, C. (2006). Walang nagbago: My affair with Marcus Adoro and the Eraserheads. In M. Calimag and J. Garcia (Comp.), Tikman ang langit: An anthology of the Eraserheads (pp. 33-37). Visual Print.

Mangulabnan, E., R. P. Marasigan, and H. A. Zabala. (1996). Fruit fairy. On Fruitcake. Greater East Asia Music and BMG Records Pilipinas.

Marasigan, R. P. (1995). Yoko. On Cutterpillow. Musiko Records and BMG Records Pilipinas.

McCoy, A. W. (1999). Closer than brothers: Manhood at the Philippine Military Academy. Yale University Press.

Mendoza, L. K. (2017). Gloc-9 as organic public intellectual: Hip and polished, raw and cool. Plaridel: $A$ Philippine Journal of Communication, Media, and Society, 14(2), 25-48.

Navarro, R. C. (2014). Musika at Bagong Lipunan: Pagbuo ng lipunang Filipino, 1972-1986. Ateneo de Manila University Press.

Navarro, R. C. (2015). Gay lingo: Pagsusuri ng "lengguwahe" sa dekada nobenta. In R.B. Tolentino and G.C. Devilles (Eds.), Kritikal na espasyo ng kulturang popular (pp. 296-326). Ateneo de Manila University Press.

Pastor, P. (2015, February 21). Before the Eraserheads became rock stars, they wrote a Pinoy musical. Philippine Daily Inquirer, D1, D4.

Perez, I.C. (2018, May 12). 'Ang Huling El Bimbo'-Why it's using Eheads songs, but can't be called an Eheads musical. Philippine Daily Inquirer, C2.

Pollisco, A. (2012). Sirena. On MKNM: Mga Kuwento ng Makata. Universal Records.

Pollisco, A. (2013). Magda. On Liham at Lihim. Universal Records.

Reyes, D. \& Manahan, J. (1992). Home Along Da Riles. In RVQ Productions. Quezon City: ABS-CBN.

Reyes, J. J. \& Manahan, J. (1991). Abangan ang Susunod na Kabanata. In ABS-CBN. Quezon City: ABS-CBN. Reyes, J. J. \& Reyes, J. J. (1995). Pare ko. Quezon City: Star Cinema. 
San Juan, D. M. (2019). Euphoria, pakikibaka, at bahala na: Kontekstuwalisasyon ng piling awiting postEDSA. Plaridel: A Philippine Journal of Communication, Media, and Society, 16(1), 39-72.

Scott, J.C . (1990). Domination and the arts of resistance: Hidden transcripts. Yale University Press.

Serquiña Jr, O. T. (2019). Manning the world: Staging Filipino migrant masculinities in Katas ng Saudi. Humanities Diliman, 16(1), 24-54.

Tolentino, R. B. (2000). Richard Gomez at ang mito ng pagkalalake, Sharon Cuneta at ang perpetwal na birhen at iba pang sanaysay ukol sa bida sa pelikula bilang kultural na texto. Anvil.

Tolentino, R. B. (2001). Sa loob at labas ng mall kong sawi / Kaliluha'y siyang nangyayaring hari: Ang pagkatuto at pagtatanghal ng kulturang popular. University of the Philippines Press.

Tolentino, R. B. (2012). Marcos, Brocka, Bernal, city films, and the contestation for imagery of nation. Kritika Kultura, 19, 115-138.

Valle, J. (2000, May 6). The Eraserheads have become better as musicians and persons. Philippine Daily Inquirer, G2.

Wild, P. H. (1969). Flower power: A student's guide to pre-hippie transcendentalism. The English Journal, $58(1), 62-68$. 


\section{Grant Support Details}

Author Contributions: All research activities and writing were done by M. Pante. The author has read and agreed to the published version of the manuscript.

Funding: The author received no specific funding for this work.

Acknowledgements: The author acknowledges the support from the University Research Council of the Ateneo de Manila University, which granted his status as a Loyola Schools research and creative work (RCW) faculty.

Conflict of Interest: The author declares no conflict of interest.

\section{About the Author \\ Si MICHAEL D. PANTE ay kawaksing propesor sa Kagawaran ng Kasaysayan, Pamantasang Ateneo de Manila, at katuwang na patungot ng Philippine Studies: Historical and Ethnographic Viewpoints. Siya ang may-akda ng librong A Capital City at the Margins: Quezon City and Urbanization in the Twentieth- Century Philippines (Ateneo de Manila University Press; Kyoto University Press, 2019), isang pagsusuri sa kasaysayan at heograpiya ng Quezon City bilang dating kabisera ng Pilipinas. (corresponding author: mpante@ateneo.edu)}


